\title{
Ovarian Cancer and Primary Peritoneal Carcinoma cM1 TNM Finding v7
}

National Cancer Institute

\section{Source}

National Cancer Institute. Ovarian Cancer and Primary Peritoneal Carcinoma CM1 TNM

Finding v7. NCl Thesaurus. Code C89663.

Ovarian cancer or primary peritoneal carcinoma with distant metastasis (excludes peritoneal metastasis). (from AJCC 7th Ed.) 